\title{
Acute Kidney Injury in the Elderly: Epidemiology, Risk Factors and Outcomes
}

\section{Antonio Del Giudice ${ }^{1 *}$, Matteo Piemontese ${ }^{1}$, Giuseppe Valente ${ }^{1}$, Michele Prencipe ${ }^{1}$, Chiara Di Giorgio ${ }^{2}$ and Filippo Aucella ${ }^{1}$}

${ }^{1}$ Division of Nephrology and Dialysis, IRCCS Casa Sollievo della Sofferenza, San Giovanni Rotondo, Italy

2Proof-reader, Department of Emergency and Organ Transplantation, University Hospital of Bari, Bari, Italy

\begin{abstract}
Structural and functional changes affecting the aging kidney predispose to an increased risk of Acute Kidney Injury (AKI) in the elderly, a condition which is becoming more and more relevant with the increase in life expectancy. The epidemiology of $\mathrm{AKI}$ in the elderly is not well assessed, because of the variable etiology, the coexistence of several comorbidities, the various clinical settings and geographical areas where the condition is managed, and the lack of uniform definition criteria. Currently, the use of the term AKI is suggested to mean any abrupt reduction in kidney function, while acute renal failure is just meant to indicate severe dysfunctions requiring renal replacement treatment. Comorbidities, common among elderly patients and several age-related conditions are risk factors for AKI. Moreover, also in elderly patients the presence of baseline proteinuria and reduced glomerular filtration rate are both powerful independent risk factors for AKI. Elderly patients with Chronic Kidney Disease (CKD) who develop AKI are at high risk for mortality, non-recovery from AKI and progression to more advanced stages of CKD and even to endstage renal disease. As a consequence, the challenge for nephrologists is to find strategies to either prevent AKI or prevent the transition from AKI to CKD.
\end{abstract}

Keywords: Acute kidney injury; Elderly

\section{Introduction}

The elderly are the fastest growing age group of the general population. In Western Europe and in the United States, the number of subjects aged $>60$ years is projected to rise from 231 million in 2000 to 395 million in 2050 [1]. Recently, also in China a continuous growth of people older than 70 has been reported as a consequence of the increase in life expectancy [2].

Epidemiological data confirm that, as an effect of improvements in life expectancy and of aging population, the median age of patients suffering from Acute Kidney Injury (AKI), formerly indicated as Acute Renal Failure (ARF), is increasing too [3].

Therefore, in the elderly, the increasing incidence of AKI is higher worldwide and is a concern that is becoming more and more relevant. For example, in China, patients aged 80 years or older are the segment of population in which the incidence of AKI has recently been reported to increase most rapidly [2]. This has induced some authors to investigate the real proportion of the problem and whether the true epidemic in renal disease is AKI or Chronic Kidney Disease (CKD) [46]. Patients with AKI who are admitted in Intensive Care Units (ICUs) have a longer length of stay, higher hospital mortality and, in case of surviving, a 5-20\% risk of dialysis-dependence [4]. In elderly patients, both CKD and AKI are often associated, but the recovery of kidney function is less complete than in patients affected by AKI alone [4].

While incidence of AKI has been progressively increasing over the recent past years, with older age, male gender and black race being associated conditions [7], its epidemiology in the elderly is far from being well assessed, due to the variable etiology, the coexistence of several comorbidities, the various clinical settings and geographical areas where the condition is managed, and the lack of uniform definition criteria [6].

This lack of standardized definitions and classification may have produced an incomplete understanding of the natural history of AKI and of the interactions between the kidney and other organ system failure [8].

\section{Structural and Functional Changes Occurring in the Aging Kidney}

The anatomical and physiological changes that occur in the kidneys with advancing age are not only the consequences of normal organ senescence but also of specific diseases, such as atherosclerosis or diabetes mellitus, which occur with greater frequency in the elderly [9].

The structural changes occurring in the normal aging of the kidney are listed in box 1 [10]

In particular, the concept of nephrosclerosis in the aging kidney has been recently reviewed by Glassock and Rule [9]. It is hypothesized that fibro-intimal hyperplasia occurring with aging in small arteries leads to glomerulosclerosis, followed by local tubular atrophy and interstitial fibrosis, and that such a constellation of findings contributes to the development of nephrosclerosis [11]. Nephrosclerosis, defined by 2 or more of these abnormalities, has been found to progressively increase with age: $2.7 \%$ for 18 to 29 -year-olds, $16 \%$ for 30 to 39 -yearolds, $28 \%$ for 40 to 49 -year-olds, $44 \%$ for 50 to 59 -year-olds, $58 \%$ for 60 to 69 -year-olds, and $73 \%$ for 70 to 77 -year-olds.

The sclerotic glomeruli of aging kidneys are smaller than the remaining functional glomeruli, in which a compensatory hypertrophy develops; both an increase in the proportion of small sclerosed glomeruli and an increase in the size of functional glomeruli may occur with age [12].

*Corresponding author: Antonio Del Giudice, MD, Division of Nephrology and Dialysis, IRCCS Casa Sollievo della Sofferenza, Viale Cappuccini, 71013 San Giovanni Rotondo, Italy, Tel: +390882410477; Fax: +390882410944; E-mail: a.delgiudice@operapadrepio.it

Received September 21, 2012; Accepted December 28, 2012; Published December 31, 2012

Citation: Del Giudice A, Piemontese M, Valente G, Prencipe M, Di Giorgio C et al. (2012) Acute Kidney Injury in the Elderly: Epidemiology, Risk Factors and Outcomes. J Nephrol Therapeut 2: 129. doi:10.4172/2161-0959.1000129

Copyright: (ㅇ 2012 Del Giudice A, et al. This is an open-access article distributed under the terms of the Creative Commons Attribution License, which permits unrestricted use, distribution, and reproduction in any medium, provided the original author and source are credited. 


Parenchyma: reduced total mass
Glomeruli: decreased epithelial cells, increased mesangial cells, sclerosis
Afferent arterioles: hyalinosis
Glomerular capillaries: atrophy
Proximal tubules: decreased number and length
Distal tubules: diverticula and cysts
Interstitium: fibrosis
Arterial vessels: atherosclerosis and constriction

Box 1: Structural changes occurring in aging kidney [10].

Conversely, the functional changes occurring in aging kidney are the reduction of renal blood flow, the declining Glomerular Filtration Rate (GFR) and ultra-filtration coefficient, the reduction of both urine concentration and dilution capacity and sodium handling [10].

Such changes progressively alter renal sensitivity to vasoconstrictors and vasodilator agents, producing an imbalance of the two processes in which there may be a role for accumulation of endogenous inhibitor of nitric oxide synthetase asymmetric dimethylarginine (ADMA) [13] and reducing autoregulatory capacity and functional reserve $[6,14,15]$. This explains the increased risk of AKI in the elderly [2], especially in hypertensives [16], in which a faster decline of creatinine clearance with age has previously been reported [17].

\section{Current Definitions of Acute Renal Failure and Acute Kidney Injury}

Because of the problems due to the use of the serum creatinine, until recently there has been no general consensus on the definition of acute renal failure [18]. The poor accuracy of the serum creatinine in reflecting the actual GFR in case of non-steady state may explain this lack of consensus and the existence of more than 30 different definitions of acute renal failure in the literature. This great variety of definitions created much confusion and made it difficult to compare results from the several studies in this area. In 2004, the Acute Dialysis Quality Initiative (ADQI) group proposed the Risk, Injury, Failure, Loss, End-stage renal disease (RIFLE) criteria for diagnosis and stratification [19]. The RIFLE criteria refer to increasing degrees of renal function loss (defined on the basis of relative increases in serum creatinine from baseline or duration and severity of oliguria) and clinical outcome measures (persistent acute renal failure) to develop a consensus definition and classification of acute renal failure [10]. However, such stratification has some limitations, as those reported in the NEiPHROS-AKI survey [20] where the serum creatinine criteria strongly predicted ICU mortality, whereas the urine output did not [18]. The existence of these limitations moved the Acute Kidney Injury Network (AKIN) to propose several refinements to the RIFLE criteria, with the addition of the words 'Acute Kidney Injury' (AKI) to represent the entire spectrum of acute renal failure and with the addition of an absolute increase in serum creatinine of $\geq 0.3 \mathrm{mg} / \mathrm{dL}$ and a 48 -hour period as the time over which the decline in kidney function has to occur $[3,21]$. AKIN proposed 3 different stages for AKI, related to the RIFLE criteria: stage 1 (Risk), stage 2 (Injury) and stage 3 (Failure), and removed RIFLE levels of Loss and End-stage renal disease, considered as outcomes rather than stages.

Currently, the use of the term Acute Kidney Injury (AKI) is suggested to mean any abrupt reduction in kidney function, while the meaning of Acute Renal Failure (ARF) is restricted to indicate severe dysfunctions requiring renal replacement treatment or other supportive interventions [3].

\section{Epidemiology}

The epidemiology of acute kidney injury around the world is still widely undefined because of the different criteria used for ascertainment and the various clinical settings and geographical areas where the condition is managed. In developed countries, in which elderly patients predominate, the epidemiology of AKI differs from that of developing areas [7].

Reported incidences of AKI in the elderly also vary according to the population studied, namely community, hospitalized or ICU patients.

In the USA, an incidence of AKI of $3.1 \%$ has been reported in a cohort of 233,803 elderly hospitalized patients evaluated in 2000 [22] and of $2.38 \%$ among Medicare beneficiaries of hospital discharges between 1992 and 2001 [7]. Among the patients of the communitybased cohort of the Kaiser Permanente of Northern California, the incidence of non-dialysis requiring AKI was found to increase from 78 per 100,000 person-years in patients aged $<50$ to 3,545 per 100,000 person-years in patients aged 80 or more $[3,23]$.

In Scotland, an incidence of AKI of 1,811 cases per million populations and an incidence of acute-on-chronic renal failure (ACRF) of 336 per million populations, respectively, have been reported; noteworthy, the median age was 76 years for AKI and 80.5 years for ACRF [24]

In some studies carried out in Spain, an overall incidence of AKI of 209 cases per million population was reported [25], with an incidence of AKI 3.5 times higher in hospitalized patients older than 70 years than in younger patients and 5 times higher in those older than 80 years than in younger ones [26].

In Italy, an incidence of AKI 10 times higher among hospitalized patients aged 65 years or more that in younger counterpart has been reported [27].

In 2010, Fang et al. [28] reported an incidence rate of AKI of $4.10 \%$ in Chinese patients aged 60 to 80 years and of $6.17 \%$ in patients aged $>80$ years who were admitted to a University Hospital in Shangai.

More recently, Wen et al. [2] reported an incidence of AKI of 2.76\% in patients aged 65 to 80 years and of $14.8 \%$ in patients aged $\geq 80$ years who had been admitted to a tertiary metropolitan hospital in Beijing.

With regard to the epidemiology of AKI in ICU patients, in the BEST Kidney study, $5.7 \%$ of 29,269 critically ill patients had ARF during their ICU stay, and $4.3 \%$ were treated with Renal Replacement Therapy (RRT). Overall hospital mortality was $60.3 \%$, and among survivors dialysis dependence at discharge was $13.8 \%$; noteworthy, the median age of the patients with ARF was 67 years [29].

In the NEFROINT study, a prospective, observational, multicenter study designed to evaluate all incident admissions in 10 ICUs, the median age was 66 years; $42.7 \%$ of 576 patients evaluated had AKI 


\begin{tabular}{|l|c|l|}
\hline Authors & Reference & Incidence \\
\hline Wen et al., China & {$[2]$} & $2.76 \%$ (aged 65-80) $14.8 \%$ (aged $>80)$ \\
\hline Xue et al., USA & {$[7]$} & $1.85 \%$ (aged <65 ) $2.86 \%$ (aged $>85)$ \\
\hline Ishani et al., USA & {$[22]$} & $3.1 \%$ \\
\hline Hsu et al., USA & {$[23]$} & $\begin{array}{l}78 / 100,000 \text { person-yrs (aged }<50) \\
3,545 / 100,000 \text { person-yrs (aged } \geq 80)\end{array}$ \\
\hline Ali et al., Scotland & {$[24]$} & 1,811 cases per million population \\
\hline Liano and Pascual, Spain & {$[25]$} & 209 cases per million population \\
\hline Pasqual et al., Spain & {$[26]$} & $\begin{array}{l}3.5-\text { times higher (aged }>70) \\
5 \text {-times higher (aged }>80)\end{array}$ \\
\hline Baraldi et al., Italy & {$[27]$} & 10 times higher in aged $\geq 65$ vs. $\leq 65$ \\
\hline Fang et al., China & {$[28]$} & $4.10 \%$ (aged $60-80) 6.17 \%$ (aged $>80)$ \\
\hline Uchino et al., multinational & {$[29]$} & $5.7 \%$ \\
\hline Garzotto et al., Italy & {$[30]$} & $65,8 \%$ \\
\hline
\end{tabular}

Table 1: Incidence of acute kidney injury (AKI) in the elderly.

within 24 hours of ICU admission, while 23\% developed new AKI later during their ICU stay [30].

Data on incidence of AKI from major studies are summarized in table 1.

\section{Risk Factors for Acute Kidney Injury: Role of Comorbidities and Age-Related Factors}

Comorbidities are common among elderly patients [6]. In an analysis conducted in 1999 on a sample of 1,217,103 Medicare beneficiaries aged 65 and older living in the US, $65 \%$ of the participants were found to have 2 or more chronic conditions, $43 \%$ had 3 or more chronic conditions and $24 \%$ had 4 or more chronic conditions [31].

Comorbidities and age-related factors can be summarized as follows: structural and functional changes in the kidney, multiple chronic comorbid conditions (CKD, cardiovascular disease, diabetes mellitus, abnormal lipid levels, vitamin D deficiency, sepsis, malnutrition), medication-related toxicity (non-steroidal anti-inflammatory drugs, diuretics, angiotensin-converting enzyme inhibitors, angiotensin receptor blockers, radiocontrast agents, nephrotoxic antibiotics), oxidative stress (with the following increase in reactive oxygen species, free radicals, and advanced glycation end products), hypovolemia and arterial hypotension, surgery (type of operation, duration of cardiopulmonary bypass) [32].

Currently, in the elderly other age-related factors, such as the telomere shortening [33], the Dicer and altered expression of specific associated microRNAs [34], the relationship between autophagy and heme-oxygenase-1 [35], Klotho deficiency [36], are thought to be involved in mechanisms predisposing to or modulating recovery from AKI [6].

\section{Risk Factors for Acute Kidney Injury: Role of Proteinuria and Reduced Glomerular Filtration Rate}

In 2008, Hsu et al. [37] compared 1,764 hospitalized adult members of Kaiser Permanente of Northern California who developed dialysisrequiring acute renal failure (mean age at hospitalization: $65.4 \pm 14.1$ years) with 600,820 hospitalized members who did not (mean age: 57.3 \pm 17.2 years). The adjusted Odds Ratios (ORs) increased significantly and progressively from 1.95 to 40.07 for patients with stages 3 - 5 of CKD compared to patients with estimated GFR in stages $1-2$ of CKD. Pre-admission baseline Diabetes Mellitus (DM), hypertension and proteinuria were also independent risk factors for AKI. In particular, patients with DM were at higher risk of AKI compared with their counterparts without DM in the same GFR category. The study showed that the propensity to develop in-hospital AKI is another complication of CKD and that the risk markedly increases even in the upper half of stage 3 estimated GFR.

In 2010, Huang et al. [38] reported the results of a prospective analysis carried on a cohort of adult patients (mean age $65.7 \pm 11.0$ years) in Taiwan who underwent Coronary Artery Bypass Grafting (CABG) between 2003 and 2007. Proteinuria, measured with a dipstick, was defined as mild (trace to $1+$ ) or heavy (2+ to $4+$ ). Among a total of 1,052 patients, cardiac surgery-associated acute kidney injury (CSA-AKI) developed in 183 patients $(17.4 \%)$ and required Renal Replacement Therapy (RRT) in 50 patients (4.8\%). In a multiple logistic regression model, mild and heavy proteinurea were each associated with an increased Odds Ratio (OR) of CSA-AKI, independent of CKD stage and the presence of DM (mild, OR 1.66; heavy, OR 2.30). Heavy proteinuria was also associated with increased OR of postoperative RRT (OR 7.29). Thus, preoperative proteinuria could be considered a predictor of CSA-AKI among patients undergoing CABG.

In 2010, Grams et al. [39] prospectively analyzed a cohort of 11,200 participants in the Atherosclerosis Risk in Communities (ARIC) study for the association between baseline urine albumin-to-creatinine ratio (UACR) and estimated GFR (eGFR) with hospitalizations or death with AKI. During the 8.0 years of the average follow-up period, the incidence of AKI was 4.0 per 1000 person-years and hospitalizations for AKI were 492 (2.8\% of a total of 17,265 hospitalizations). Noteworthy, the mean age of the AKI-patients was 64.7 years vs. 62.8 years of non AKIpatients. Using participants with UACRs $<10 \mathrm{mg} / \mathrm{g}$ as a reference, the relative hazards of AKI, adjusted for age, gender, race, cardiovascular risk factors and categories of eGFR were 1.9, 2.2, and 4.8 for UACR groups of 11 to $29 \mathrm{mg} / \mathrm{g}, 30$ to $299 \mathrm{mg} / \mathrm{g}$ and $\geq 300 \mathrm{mg} / \mathrm{g}$, respectively. Similarly, the overall adjusted relative hazard of AKI increased with decreasing eGFR. Therefore, these data from a large, prospectively followed population-based cohort demonstrated that UACR and eGFR exhibit a strong, independent and graded association with incidence of AKI, also in older patients.

A similar association of proteinuria and GFR with AKI was reported in 2010 by James et al. [40] who used a large sample $(920,985)$ of adults in Alberta, Canada, $10 \%$ of whom with a mean age of 67 to 78 years. The aim was to investigate how eGFR and proteinuria jointly modified the risk of AKI and of subsequent adverse clinical outcomes. During a median follow-up of 35 months, 6,520 participants $(0.7 \%)$ were admitted with AKI and $615(0.06 \%)$ with AKI requiring dialysis. Patients with normal eGFR values $\left(\geq 60 \mathrm{~mL} / \mathrm{min}\right.$ per $\left.1.73 \mathrm{~m}^{2}\right)$ and mild proteinuria (urine dipstick trace to $1+$ ) had 2.5 times the risk of hospital admission with AKI than did patients with no urinary protein; this risk was 4.4-fold increased for patients with heavy proteinuria (urine dipstick $\geq 2+$ ). The authors concluded that the risk of admittance to hospital for patients with AKI increased with heavier proteinuria and reduced eGFR.

In 2011, Hsu and Hsu [41] reviewed the above 4 studies and stated that, on the whole, these data confirm that also in older patients the presence of baseline proteinuria and reduced GFR are both powerful independent risk factors for AKI, that even a very mild degree of proteinuria predicts increased risk of AKI, that the risk of AKI increases along the severity of baseline proteinuria, and that there is a graded association between reduced eGFR at baseline and risk of AKI, independently of proteinuria.

\section{Mortality}

Acute kidney injury is associated with an extremely high mortality 
rate, ranging from $37 \%$ [42] to $60 \%$ [30], with higher rates having been reported in critical care settings instead of non-critical care settings, as a possible consequence of multiorgan failure seen in the former. Furthermore, while declining rates in overall mortality have been recently reported [43], data on mortality in the elderly are conflicting $[7,22-25,27,42-46]$.

With regard to the risk of long-term mortality after an episode of AKI, in their study on the cohort of 233,803 elderly hospitalized patients assessed in 2000, Ishani et al. [22] reported a 2-year combined mortality rate of $29.1 \%$ that was higher in patients with AKI and CKD than in those with AKI alone.

Lo et al. [42] studied the outcomes of 562,779 patients of Kaiser Permanente of Northern California who were hospitalized over an 8-year period and whose mean age at admission was $63.5 \pm 14.8$ years. The authors reported an in-patient death of $41.9 \%$ in subjects with dialysis-requiring $\mathrm{ARF}$ and of $1.14 \%$ in those with non-dialysisrequiring ARF; moreover, they found that, over the 6 years of followup an episode of dialysis-requiring ARF was independently associated with a more than two-fold increased long-term risk of death. However, such an increase in the risk of long-term mortality following AKI was not observed by Wald et al. $[45,46]$.

AKI should now be recognized as a potent predictor not only of inhospital mortality [47] but also of long-term morbidity and mortality [48]. Patients who, after admission to hospital, are discharged with AKI are 3 times more likely to die and 13 times more likely to reach ESRD in the year after hospital admission than patients who are admitted to hospital without AKI [49].

The increased mortality risk associated with AKI persists over time and after adjustment for post-discharge eGFR $[40,50]$. In the study carried out by James et al. [40], 27,959 of 920,985 participants (3\%) died during the follow-up. Rates of all-cause mortality were higher in patients after hospital admission with AKI than in those without AKI. The prognostic significance of AKI varied according to baseline eGFR; however, heavy proteinuria was responsible for the high rates of death in participants with AKI and baseline eGFR $\geq 60 \mathrm{~mL} / \mathrm{min}$ per $1.73 \mathrm{~m}^{2}$. The authors concluded that long-term mortality increases after AKI at all levels of eGFR and proteinuria. However, they suggest that the risk of adverse outcomes attributable to AKI is not constant for all categories of baseline kidney function. In their study, the prognostic importance of AKI decreases as pre-existing kidney damage worsens. In other words, an AKI event in people with normal kidney function greatly increases their subsequent risk of ESRD and death; in people with stage 4 of CKD, an event of AKI adds a lower risk, but the baseline risk of adverse events is increased.

Therefore, it is believed that, on average, the severity of illness that is necessary to provoke AKI in a person with normal kidneys is greater than that required to provoke injury in a person with pre-existing kidney damage [51].

The role of only a modestly reduced eGFR in determining outcomes in aging subjects has been now been clarified as a result of large observational studies and meta-analyses [52]. The results show that younger patients with an eGFR of $45-59 \mathrm{ml} / \mathrm{min}$ per $1.73 \mathrm{~m}^{2}$ are at increased risk of death, usually due to cardiovascular disease. This effect is softened by advancing age; therefore, older patients with an eGFR of $45-59 \mathrm{ml} / \mathrm{min}$ per $1.73 \mathrm{~m}^{2}$ seem not to be at increased risk of death after correction for age [53,54]. Moreover, the existence of a ' $U$-shaped' effect, in which mortality is elevated in both higher and lower strata of eGFR, can be hypothesized $[40,52,55]$.
Recently, Gong et al. [56] conducted a prospective study on 99 consecutive Chinese patients with AKI, aged 65 to 96 years (mean 77.8 \pm 7.8 years), with the aim of investigating the clinical features and risk factors affecting mortality. The patients were divided into survivors and non-survivors, according to their outcomes, and factors including clinical characteristics and laboratory features were compared between the 2 groups. Significant differences between the 2 groups were found in concomitant diseases, Multiple Organ Dysfunction Syndrome (MODS), albumin, C-reactive protein and prealbumin levels. The mortality rate of the elderly patients with AKI was $42 \%$ vs. $24 \%$ of the non-elderly group. The clinical condition of 41 elderly AKI patients was complicated by the presence of MODS and 20 died of MODS. An increase in mortality rate from $39 \%$ to $100 \%$, depending on the number of failed organs, was observed: $39 \%$ in patients with 2 organ dysfunctions, $50 \%$ in patients with 3 organ dysfunctions, $60 \%$ in patients with 4 organ dysfunctions, and $100 \%$ in patients with 5 organ dysfunctions. In the multivariate logistic regression analysis, concomitant disease $(\mathrm{p}=0.003)$ and MODS $(\mathrm{p}=0.001)$ were found to be independent risk factors for death of the elderly patients with AKI after adjusting for age, sex, pre-albumin, sepsis/infection, and serum creatinine.

The presence of multiorgan failure had already been reported by Van Den Noortgate et al. [1] as one of the variables predicting inhospital mortality in elderly patients with acute renal failure requiring dialysis post-cardiac surgery. Surprisingly, in this study, age was not found to influence the overall outcome both in older patient and in younger patients. According to the authors, this relatively beneficial outcome in the elderly might be the consequence of a different severity of the underlying disease. On the one hand, the aging kidney is less able to adapt to rapid hemodynamic changes and electrolyte balance, so AKI might develop more easily in the older patients. On the other hand, in the elderly, comorbid factors that are at the origin of AKI might be less preponderant or severe than in the younger population. After all, comorbid conditions probably more frequently lead to a fatal outcome than renal failure by itself.

With regard to malnutrition, the results of a meta-analysis of 17 observational clinical studies carried out by Wiedermann et al. [57] are noteworthy. Of the 17 studies, 11 evaluated the influence of serum albumin on AKI incidence and 6 evaluated the relationship between serum albumin and mortality among patients who developed AKI. In 3,917 patients evaluated, a substantial number of whom were aged 65 or more, the authors found that the lower serum albumin was an independent predictor both of AKI and of death after AKI development.

More recently, Oliva et al. [58] reported that low serum albumin is an independent predictor of mortality in Spanish chronic hemodialysis patients of over 75 years old.

In the older group with AKI evaluated by Wen et al. [2], MODS, heart failure and gastrointestinal bleeding were found to be independent risk factors for the 90-day mortality, while the presence of MODS, malnutrition, gastrointestinal bleeding, absolute increase in serum creatinine and the use of alfa-ketoacid were found to be independent variables predicting medium-term (at 1 year) survival/mortality. If classified according to the AKIN categories, patients with AKI stage 2 and 3 exhibited a significantly worse survival than patients with AKI stage $1[59,60]$. Comparing the 201 very old patients with de novo AKI (42.5\%) and the 272 very old patients with AKI superimposed on CKD 


\begin{tabular}{|c|c|c|}
\hline Authors & Reference & Mortality \\
\hline Wen et al., China & [2] & $\begin{array}{l}46.27 \% \text { (at } 90 \text { days, de novo } \\
\text { AKI) } \\
29.04 \% \text { (at } 90 \text { days, acute- } \\
\text { on-chronic kidney injury) }\end{array}$ \\
\hline Xue at al., USA & [7] & $\begin{array}{l}37.8 \% \text { (in-hospital) } \\
34.5-48.6 \% \text { (90 days) }\end{array}$ \\
\hline Ishani et al., USA & [22] & $29.1 \%$ (2 years) \\
\hline Ali et al., Scotland & [24] & $\begin{array}{l}32.7 \% \text { (in-hospital) } \\
41.4 \% \text { (90 days) } \\
49.8 \% \text { ( } 6 \text { months) }\end{array}$ \\
\hline Baraldi et al., Italy & {$[27]$} & $\begin{array}{l}33.3 \%(\text { aged } \geq 65) \\
2.5 \%(\text { aged } \leq 64)\end{array}$ \\
\hline Ukino et al., multinational & [29] & $60.3 \%$ \\
\hline Garzotto et al., Italy & [30] & $\begin{array}{l}21.7 \% \text { (intensive care unit } \\
\text { and in-hospital) }\end{array}$ \\
\hline James et al., Canada & [40] & $3 \%$ at 35 months \\
\hline Lo et al., USA & [42] & $\begin{array}{l}41.9 \% \text { (dialysis-requiring } \\
\text { acute renal failure) } \\
1.14 \% \text { (non-dialysis-requiring } \\
\text { acute renal failure) }\end{array}$ \\
\hline Gong et al., China & {$[56]$} & $42 \%$ \\
\hline Sesso et al., Brazil & [59] & $\begin{array}{l}41 \% \text { (in-hospital) } \\
59 \% \text { (community) }\end{array}$ \\
\hline Kohli et al., India & {$[60]$} & $60.9 \%$ \\
\hline
\end{tabular}

Table 2: Mortality from acute kidney injury (AKI) in the elderly.

(acute-on-chronic, A-on-C kidney injury) (57.5\%), the former were found to be less old and to exhibit a higher percentage increase in serum creatinine and a higher mortality rate within 90 days $(46.27 \%$ vs. $29.04 \%$ ). This mortality rate was higher than the rate of $20-40 \%$ reported in previous reports on patients older than 65 years, and confirms age being a risk factor for in-hospital mortality in patients of this range of age.

Data on mortality for AKI are summarized in table 2.

\section{Non-Recovery from Acute Kidney Injury}

For a long time acute kidney injury has been considered a completely reversible syndrome. However, data from recent studies conducted on animals and humans indicate that AKI more than likely results in a permanent renal damage and can also affect other organs [61-63].

Elderly patients with CKD are at high risk of non-recovery from $\mathrm{AKI}$ and for progression to more advanced stages of CKD or even to ESRD [6].

In the study carried out by James et al. [40], 771 of 920,985 participants $(0.08 \%)$ developed End-Stage Renal Disease (ESRD) during the follow-up, and 2,341 of 580,452 (0.4\%) had a doubling of serum creatinine values. The authors concluded that the risk of clinically relevant kidney function loss was higher after AKI.

In a systematic review and meta-analysis of 17 studies published between 2000 and 2007, Schmitt et al. [62] reported data on recovery of kidney function by age and showed that in $31.3 \%$ of the surviving elderly patients kidney function did not recover, while it did in $26 \%$ of the younger patients (pooled relative risk, 1.28, $\mathrm{p}<0.05$ ).

In the cohort of 233,803 hospitalized elderly patients evaluated by Ishani et al. [22], 3.1\% survived to discharge with a diagnosis of AKI and 5.3 per 1000 developed ESRD; after adjustment for age, gender, race, DM and hypertension, the hazard ratio for developing ESRD was 41.2 for patients with AKI and CKD relative to those without kidney disease, 13.0 for patients with AKI and without previous CKD, and 8.4 for patients with CKD and without AKI. Thus, elderly patients with $\mathrm{AKI}$, in particular those with previously diagnosed CKD, were reported to be at a significantly increased risk for ESRD, suggesting that episodes of AKI may accelerate progression of renal disease.

Therefore, large population-based studies have demonstrated that patients who survive an episode of AKI are at a considerable risk of progressing to advanced stages of CKD. The same studies demonstrate the continuity of disease from AKI to CKD to End-Stage Renal Disease (ESRD). In particular, Amdur et al. [63], using a US Department of Veterans Affairs database to ascertain the long-term renal function in 113,272 patients, showed that up to $20 \%$ of the patients with an inpatient diagnosis of Acute Tubular Necrosis (ATN) progressed to CKD stage 4 or greater within 18-24 months. However, within this population, a subset of patients appeared to nearly achieve a total recovery and not to progress to advanced stages of CKD. Therefore, it is likely that some patients who develop AKI are at higher risk for CKD progression than others.

Chawla et al. [64] hypothesized that patients surviving AKI who are at higher risk for progression to CKD can be well characterized. In order to identify and stratify AKI patients who are more likely to progress to $\mathrm{CKD}$, the authors developed a prediction tool that was assessed on 5,351 elderly patients (mean age, $66.3 \pm 12.3$ years) hospitalized in the US Department of Veterans Affairs Healthcare System with a primary diagnosis of AKI. Especially those patients with AKI who required dialysis and then recovered were at high risk for progression to CKD. Hence, the severity of AKI was believed to be a robust predictor of progression to CKD. Noteworthy, among the demographic variables, age was associated with progression to CKD stage 4 , with each year of age raising the odds ratio (OR) of entering CKD by $1 \%$ in univariate prediction model and by $1-2 \%$ in multivariate prediction models. Thus, advanced age could be useful to identify patients at risk for progression to CKD after an episode of AKI.

More recently, Coca et al. [61] conducted a systematic review and meta-analysis of 13 selected studies evaluating long-term renal and non-renal outcomes in patients with $\mathrm{AKI}$, in order to estimate the risk for CKD, ESRD, death and other non-renal outcomes in patients with AKI vs. those without AKI, and in order to determine whether preexisting renal injury, such as decreased baseline GFR or pre-existing proteinuria, modified these associations. Definitions of AKI varied substantially: need for RRT $[42,45,65]$, increase in plasma creatinine of $0.3-0.5$ or $0.6-3.0 \mathrm{mg} / \mathrm{dL}$, ARF and acute tubular necrosis (ATN) [63]. Severity of AKI was graded as class 1-4 of AKI (class 1, 1\%-24\% increase in creatinine; class 2, 25\%-49\% increase in creatinine; class 3 , $50 \%-90 \%$ increase in creatinine; class $4,>100 \%$ increase in creatinine) [66] or as mild, moderate and severe AKI. As much as 13 studies were selected for inclusion into the systematic review. Of these 13 studies, 4 were conducted on cohorts of patients aged more than 60 and 6 on cohorts of patients with more than 65 years of age.

In the studies carried out on AKI survivors older than 65 years, the following renal outcome rates and hazard ratios (HRs), at a mean follow-up of 20 to 75 months, have been reported: CKD, from 6.6-10.5 per 100 person-years for patients with mild and moderate-severe AKI, respectively, HR 2.33 [66], to 22 per 100 person-years, HR 6.64 [63]; ESRD, from 0.6 per 100 person-years, HR 3.26 [67], to 12.1 per 100 person-years, HR 2.33 [68].

Regardless of the heterogeneity of the statistical data, the results of this meta-analysis show that the risks for both CKD and ESRD, as 
well as the adjusted HRs, increased in a graded manner with mild AKI, moderate AKI and severe AKI

Change effects due to reduced baseline GFR and to proteinuria before AKI should also be considered.

In the 2 studies reporting the risk for CKD after AKI in subjects with both reduced and normal baseline GFR [40,42], the relative risk (hazard ratio, HR) for CKD was higher in patients who did not have a decreased baseline GFR (adjusted HR 38.8,) than in those with a decreased baseline GFR (adjusted HR 24.4). As already seen for mortality rate, the role of only a modestly reduced eGFR in determining the progression of CKD in aging subjects has been the subject of several investigations which showed that older patients with an eGFR of 45-59 $\mathrm{ml} / \mathrm{min}$ per $1.73 \mathrm{~m}^{2}$ are less likely than younger patients to progress to end-stage renal disease, and when progression does occur it is slower than in younger patients $[53,69]$

Similarly, in the 2 studies reporting the risk for ESRD both in patients with and without decreased baseline GFR, the relative risk for ESRD was higher in subjects with AKI who did not have decreased baseline GFR.

Moreover, in one of the two studies cited (i.e. that of James et al.) [40], patients with AKI who had a pre-existing proteinuria (and a normal baseline GFR) had a lower relative risk for CKD (defined as doubling of creatinine or ESRD) (HR 9.7) compared with patients without proteinuria (HR 30.0). Moreover, James reported the association between the different severity grades of AKI (mild, moderate and severe) and the increased occurrence of non-renal outcomes such as congestive heart failure and cardiovascular disease [40].

According to Coca et al. [61], their meta-analysis gives the opportunity to assess the absolute and relative risk associated with these outcomes (CKD and ESRD) after AKI. They consider potentially counterintuitive the fact that patients with normal GFR before AKI have a higher relative risk for the development of ESRD if compared with those developing AKI in a decreased baseline GFR setting. They cite as an example the study of Wald et al. [45] and state that, although the absolute risk of ESRD in subjects without previous AKI and without a decrease in baseline GFR was of $9.8 \%$ - while it was almost doubled (to $18.4 \%$ ) in subjects with AKI and reduced baseline GFR-, the relative risk for ESRD was higher in the subjects with normal GFR, because of the extremely low probability of ESRD in subjects without previous AKI or reduced baseline GFR [61].

In conclusion, AKI is an important cause of ESRD because it can lead to an acceleration of the normal age-related decline in GFR. Moreover, patients suffering from repeated or prolonged injuries are predisposed to $\mathrm{CKD}$ since, although tubular regeneration may occur after AKI, cellular plasticity may be lost and the inflammatory processes occurring during repair and regeneration phases can lead to paracrine stimulation of myofibroblasts. The consequent development of progressive tubulo-interstitial fibrosis accelerates progression of CKD to ESRD [70]. On the other hand of this AKI-CKD nexus [71], the reduction of the renal mass of CKD, similarly to diabetic and agingrelated pathology, predisposes patients to AKI. The literature on the reciprocal relationship between $\mathrm{AKI}$ and $\mathrm{CKD}$ in elderly patients that is the way in which these conditions predispose to each other, has been recently reviewed by Coca et al. [72]

According to the authors, the plethora of data indicating that not only does AKI often lead, but also predisposes to CKD, is a concern for public health. In fact, since approximately 40 million people in the US were aged $>65$ in 2010 , since the incidence of AKI in this elderly population is approximately 3,000 per 100,000 person-years [23], since $75 \%$ will survive to discharge after AKI, and since the incidence of stage 4 or worse of CKD after AKI is approximately 120 per 1,000 person-years [63], then approximately 100,000 elderly people per year in the US are developing new CKD after an episode of AKI. So, if is true that biomarkers are transforming our understanding of AKI [73], the challenge for the nephrology community is that of finding strategies to either prevent AKI or prevent the transition from AKI to CKD [72].

\section{References}

1. Van Den Noortgate N, Mouton V, Lamot C, Van Nooten G, Dhondt A, et al. (2003) Outcome in a post-cardiac surgery population with acute renal failure requiring dialysis: does age make a difference? Nephrol Dial Transplant 18: 732-736.

2. Wen J, Cheng Q, Zhao J, Ma Q, Song T, et al. (2012) Hospital-acquired acute kidney injury in Chinese very elderly persons. J Nephrol May 28:0. doi: 10.5301/ jn.5000182. (Epub ahead of print)

3. Abdel-Kader K, Palevsky PM (2009) Acute kidney injury in the elderly. Clin Geriatr Med 25: 331-358.

4. Hoste EA, Schurgers M (2008) Epidemiology of acute kidney injury: how big is the problem? Crit Care Med 36: S146-S151.

5. Hsu CY (2010) Where is the epidemic in kidney disease? J Am Soc Nephrol 21: $1607-1611$

6. Anderson S, Eldadah B, Halter JB, Hazzard WR, Himmelfarb J, et al. (2011) Acute kidney injury in older adults. J Am Soc Nephrol 22: 28-38.

7. Xue JL, Daniels F, Star RA, Kimmel PL, Eggers PW, et al. (2006) Incidence and mortality of acute renal failure in Medicare beneficiaries, 1992 to 2001. J Am Soc Nephrol 17: 1135-1142.

8. Himmelfarb J, Ikizler TA (2007) Acute kidney injury: changing lexicography, definitions, and epidemiology. Kidney Int 71: 971-976.

9. Glassock RJ, Rule AD (2012) The implications of anatomical and functiona changes of the aging kidney: with an emphasis on the glomeruli. Kidney Int 82: $270-277$.

10. Lautrette A, Heng AE, Jaubert D, Hssain AA, Deteix P, et al. (2012) Insuffisance rénale aiguë du sujet âgé. Néphrol Thér 8: 57-62.

11. Martin JE, Sheaff MT (2007) Renal ageing. J Pathol 211: 198-205.

12. Tsuboi N, Kawamura T, Koike K, Okonogi H, Hirano K, et al. (2010) Glomerular density in renal biopsy specimens predicts the long-term prognosis of $\lg A$ nephropathy. Clin J Am Soc Nephrol 5: 39-44.

13. Kielstein JT, Bode-Böger SM, Haller H, Fliser D (2003) Functional changes in the ageing kidney: is there a role for asymmetric dimethylarginine? Nephrol Dial Transplant 18: 1245-1248.

14. Castellani S, Ungar A, Cantini C, La Cava G, Di Serio C, et al. (1998) Excessive vasoconstriction after stress by the aging kidney: inadequate prostaglandin modulation of increased endothelin activity. J Lab Clin Med 132: 186-194.

15. Fuiano G, Sund S, Mazza G, Rosa M, Caglioti A, et al. (2001) Renal hemodynamic response to maximal vasodilating stimulus in healthy olde subjects. Kidney Int 59: 1052-1058.

16. Abuelo JG (2007) Normotensive ischemic acute renal failure. N Engl J Med 357: 797-805.

17. Lindeman RD, Tobin JD, Shock NW (1984) Association between blood pressure and the rate of decline in renal function with age. Kidney Int 26: 861-868.

18. Palewsky PM (2011) Definition of acute kidney injury (acute renal failure) UpToDate online 19.2. Available at: http://www.uptodate.com. Accessed September 27.2011

19. Bellomo R, Ronco C, Kellum JA, Mehta RL, Palevsky P; Acute Dialysis Quality Initiative workgroup (2004) Acute renal failure - definition, outcome measures, animal models, fluid therapy and information technology needs: the Second International Consensus Conference of the Acute Dialysis Quality Initiative (ADQI) Group. Crit Care 8: R204-212. 
Citation: Del Giudice A, Piemontese M, Valente G, Prencipe M, Di Giorgio C, et al. (2012) Acute Kidney Injury in the Elderly: Epidemiology, Risk Factors and Outcomes. J Nephrol Therapeut 2: 129. doi:10.4172/2161-0959.1000129

Page 7 of 8

20. Cruz DN, Bolgan I, Perazella MA, Bonello M, de Cal M, et al. (2007) North East Italian Prospective Hospital Renal Outcome Survey on Acute Kidney Injury (NEiPHROS-AKI): targeting the problem with the RIFLE Criteria. Clin J Am Soc Nephrol 2: 418-425.

21. Mehta RL, Kellum JA, Shah SV, Molitoris BA, Ronco C, et al. (2007) Acute Kidney Injury Network: report of an initiative to improve outcomes in acute kidney injury. Crit Care 11: R31.

22. Ishani A, Xue JL, Himmelfarb J, Eggers PW, Kimmel PL, et al. (2009) Acute kidney injury increases risk of ESRD among elderly. J Am Soc Nephrol 20: 223-228.

23. Hsu CY, McCulloch CE, Fan D, Ordoñez JD, Chertow GM, et al. (2007) Community-based incidence of acute renal failure. Kidney Int 72: 208-212.

24. Ali T, Khan I, Simpson W, Prescott G, Townend J, et al. (2007) Incidence and outcomes in acute kidney injury: a comprehensive population-based study. J Am Soc Nephrol 18: 1292-1298.

25. Liano F, Pascual J (1996) Epidemiology of acute renal failure: a prospective, multicenter, community-based study. Madrid Acute Renal Failure Study Group. Kidney Int 50: 811-818.

26. Pasqual J, Orofino L, Liano F, Marcén R, Naya MT, et al. (1990) Incidence and prognosis of acute renal failure in older patients. J Am Geriatr Soc 38: 25-30

27. Baraldi A, Ballestri M, Rapanà R, Lucchi L, Borella P, et al. (1998) Acute rena failure of medical type in an elderly population. Nephrol Dial Transplant 13 : 25-29.

28. Fang Y, Ding X, Zhong Y, Zou J, Teng J, et al. (2010) Acute kidney injury in a Chinese hospitalized population. Blood Purif 30: 120-126

29. Uchino S, Kellum JA, Bellomo R, Doig GS, Morimatsu H, et al. (2005) Acute renal failure in critically ill patients: a multinational, multicenter study. JAMA 94: 813-818

30. Garzotto F, Piccinni P, Cruz D, Gramaticopolo S, Dal Santo M, et al. (2011) RIFLE-based data collection/management system applied to a prospective cohort multicenter Italian study on the epidemiology of acute kidney injury in the intensive care unit. Blood Purif 31: 159-171.

31. Wolff JL, Starfield B, Anderson G (2002) Prevalence, expenditures, and complications of multiple chronic conditions in the elderly. Arch Intern Med 162 2269-2276.

32. Choudhury D, Levi M (2011) Kidney aging--inevitable or preventable? Nat Rev Nephrol 7: 706-717.

33. Westhoff JH, Schildhorn C, Jacobi C, Homme M, Hartner A, et al. (2010) Telomere shortening reduces regenerative capacity after acute kidney injury. $J$ Am Soc Nephrol 21: 327-336.

34. Wei Q, Bhatt K, He HZ, Mi QS, Haase VH, et al. (2010) Targeted deletion of Dicer from proximal tubules protects against renal ischemia-reperfusion injury. J Am Soc Nephrol 21: 756-761.

35. Bolisetty S, Traylor AM, Kim J, Joseph R, Ricart K, et al. (2010) Heme oxygenase- 1 inhibits renal tubular macroautophagy in acute kidney injury. $J$ Am Soc Nephrol 21: 1702-1712.

36. Hu MC, Kuro-o M, Moe OW (2012) The emerging role of Klotho in clinical nephrology. Nephrol Dial Transplant 27: 2650-2657.

37. Hsu CY, Ordonez JD, Chertow GM, McCullouch CE, Go AS (2008) The risk of acute renal failure in patients with chronic kidney disease. Kidney Int 74 101-107.

38. Huang TM, Wu VC, Young GH, Lin YF, Shiao CC, et al. (2011) Preoperative proteinuria predicts adverse renal outcomes after coronary artery bypass grafting. J Am Soc Nephrol 22: 156-163.

39. Grams ME, Astor BC, Bash LD, Matsushita K, Wang Y, et al. (2010) Albuminuria and estimated glomerular filtration rate independently associate with acute kidney injury. J Am Soc Nephrol 21: 1757-1764.

40. James MT, Hemmelgarn BR, Wiebe N, Pannu N, Manns BJ, et al. (2010) Glomerular filtration rate, proteinuria, and the incidence and consequences of acute kidney injury: a cohort study. Lancet 376: 2096-2103.

41. Hsu RK, Hsu CY (2011) Proteinuria and reduced glomerular filtration rate as risk factors for acute kidney injury. Curr Opin Nephrol Hypertens 20: 211-217.

42. Lo LJ, Go AS, Chertow GM, McCulloch CE, Fan D, et al. (2009) Dialysis- requiring acute renal failure increases the risk of progressive chronic kidney disease. Kidney Int 76: 893-899.

43. Waikar SS, Curhan GC, Wald R, McCarthy EP, Chertow GM (2006) Declining mortality in patients with acute renal failure, 1988 to 2002. J Am Soc Nephrol 17: $1143-1150$.

44. Yong K, Dogra G, Boudville N, Pinder M, Lim W (2011) Acute kidney injury: controversies revisited. Int J Nephrol: 762634. Epub 2011 Apr 14.

45. Wald R, Quinn RR, Luo J, Li P, Scales DC, et al. (2009) Chronic dialysis and death among survivors of acute kidney injury requiring dialysis. JAMA 302: 1179-1185.

46. Waikar SS, Winkelmayer WC (2009) Chronic on acute renal failure: long-term implications of severe acute kidney injury. JAMA 302: 1227-1229.

47. Lameire N, Van Biesen W, Vanholder R (2005) Acute renal failure. Lancet 365 417-430.

48. Coca SG, Yusuf B, Shlipak MG, Garg AX, Parikh CR (2009) Long-term risk of mortality and other adverse outcomes after acute kidney injury: a systematic review and meta-analysis. Am J Kidney Dis 53: 961-973.

49. United States Renal Data System (2009) The concise 2009 annual data report Atlas of chronic kidney disease and end-stage renal disease in the United States. http://www.usrds.org/2009/usrds_booklet_09.pdf. Accessed August 12, 2012.

50. Lafrance JP, Miller DR (2010) Acute kidney injury associates with increased long-term mortality. J Am Soc Nephrol 21: 345-352.

51. Grams M, Coresh J (2010) Proteinuria and risk of acute kidney injury. Lancet 376: 2046-2048.

52. Gansevoort RT, Matsushita K, van der Velde M, Astor BC, Woodward M, et al. (2011) Lower estimated GFR and higher albuminuria are associated with adverse kidney outcomes. A collaborative meta-analysis of general and highrisk population cohorts. Kidney Int 80: 93-104.

53. O'Hare AM, Choi Al, Bertenthal D, Bacchetti P, Garg AX, et al. (2007) Age affects outcomes in chronic kidney disease. J Am Soc Nephrol 18: 2758-2765.

54. O'Hare AM, Bertenthal D, Covinsky KE, Landefeld CS, Sen S, et al. (2006) Mortality risk stratification in chronic kidney disease: one size for all ages? J Am Soc Nephrol 17: 846-853.

55. Astor BC, Matsushita K, Gansevoort RT, van der Velde M, Woodward M, et al. (2011) Lower estimated glomerular filtration rate and higher albuminuria are associated with mortality and end-stage renal disease. A collaborative metaanalysis of kidney disease population cohorts. Kidney Int 79: 1331-1340.

56. Gong Y, Zhang F, Ding F, Gu Y (2012) Elderly patients with acute kidney injury (AKI): clinical features and risk factors for mortality. Arch Gerontol Ger 54 e47-e51.

57. Wiedermann CJ, Wiedermann W, Joannidis M (2010) Hypoalbuminemia and acute kidney injury: a meta-analysis of observational clinical studies. Intensive Care Med 36: 1657-1665.

58. Oliva JS, Roa LM, Lara A, Garrido S, Salgueira M, et al. (2012) Survival and factors predicting mortality in hemodialysis patients over 75 years old. J Nephrol 2012 Mar 29.0. doi: 105301/jn.500017. Epub ahead of print.

59. Sesso R, Roque A, Vicioso B, Stella S (2004) Prognosis of ARF in hospitalized elderly patients. Am J Kidney Dis 44: 410-419.

60. Kohli HS, Bhat A, Aravindan AN, Sud K, Jha V, et al. (2007) Predictors of mortality in elderly patients with acute renal failure in a developing country. In Urol Nephrol 39: 339-344.

61. Coca SG, Singanamala S, Parikh CR (2012) Chronic kidney disease after acute kidney injury: a systematic review and meta-analysis. Kidney Int 81: 442-448.

62. Schmitt R, Coca S, Kanbay M, Tinetti ME, Cantley LG, et al. (2008) Recovery of kidney function after acute kidney injury in the elderly: a systematic review and meta-analysis. Am J Kidney Dis 52: 262-271.

63. Amdur RL, Chawla LS, Amodeo S, Kimmel PL, Palant CE (2009) Outcomes following diagnosis of acute renal failure in U.S. veterans: focus on acute tubular necrosis. Kidney Int 76: 1089-1097.

64. Chawla LS, Amdur RL, Amodeo S, Kimmel PL, Palant CE (2011) The severity of acute kidney injury predicts progression to chronic kidney disease. Kidney Int 79: 1361-1369. 
Citation: Del Giudice A, Piemontese M, Valente G, Prencipe M, Di Giorgio C, et al. (2012) Acute Kidney Injury in the Elderly: Epidemiology, Risk Factors and Outcomes. J Nephrol Therapeut 2: 129. doi:10.4172/2161-0959.1000129

Page 8 of 8

65. Hsu CY, Chertow GM, McCulloch CE, Fan D, Ordoñez JD, et al. (2009) Nonrecovery of kidney function and death after acute on chronic renal failure. Clin J Am Soc Nephrol 4: 891-898.

66. Ishani A, Nelson D, Clothier B, Schult T, Nugent S, et al. (2011) The magnitude of acute serum creatinine increase after cardiac surgery and the risk of chronic kidney disease, progression of kidney disease, and death. Arch Intern Med 171: 226-233.

67. Newsome BB, Warnock DG, McClellan WM, Herzog CA, Kiefe Cl, et al. (2008) Long-term risk of mortality and end-stage renal disease among the elderly after small increases in serum creatinine level during hospitalization for acute myocardial infarction. Arch Intern Med 168: 609-616.

68. Lafrance JP, Djurdjev O, Levin A (2010) Incidence and outcomes of acute kidney injury in a referred chronic kidney disease cohort. Nephrol Dial Transplant 25: 2203-2209.
69. Prakash S, O'Hare AM (2009) Interaction of aging and chronic kidney disease. Semin Nephrol 29: 497-503.

70. Venkatachalam MA, Griffin KA, Lan R, Geng H, Saikumar P, et al. (2010) Acute kidney injury: a springboard for progression in chronic kidney disease. Am J Physiol Renal Physiol 298: F1078-1094.

71. Okusa MD, Chertow GM, Portilla D; Acute Kidney Injury Advisory Group of the American Society of Nephrology (2009) The nexus of acute kidney injury, chronic kidney disease, and World Kidney Day 2009. Clin J Am Soc Nephrol 4: $520-522$.

72. Coca SG, Cho KC, Hsu CY (2011) Acute kidney injury in the elderly: predisposition to chronic kidney disease and vice versa. Nephron Clin Pract 119: c19-c24.

73. Chawla LS, Kellum JA (2012) Acute kidney injury in 2011: Biomarkers are transforming our understanding of AKI. Nat Rev Nephrol 8: 68-70. 